\title{
RIEMANN'S FUNCTION HAS AN EXPONENTIAL BOUND
}

\author{
PAUL R. BEESACK
}

\begin{abstract}
The Riemann function $v(t ; x)$ of a hyperbolic characteristic initial value problem has been much used in recent years to provide upper bounds for functions which satisfy Gronwall-type integral inequalities. This note gives a direct proof of the fact that $v$ satisfies an inequality of the form $v(t ; x) \leqslant \exp \left(\int_{t}^{x} h(s) d s\right)$.
\end{abstract}

In 1972, D. R. Snow [13, 14] gave one of the first Gronwall-type integral inequalities involving two independent variables. His technique was to reduce the inequality to a second-order partial differential inequality, which he then integrated using Riemann's method. In 1973, E. C. Young [18] extended Snow's technique to the case of $n$ independent variables, and later J. Chandra and P. W. Davis [5] gave a further extension to integral inequalities involving matrix functions. Since then, the method has been often used (see, for example, $[1,3,4,7-12,15,17])$ to give upper bounds for functions $u: \mathbf{R}^{n} \rightarrow \mathbf{R}$ which satisfy integral inequalities of the form

$$
u(x) \leqslant a(x)+\int_{x^{0}}^{x} b(t) u(t) d t,
$$

where

$$
\int_{x^{0}}^{x} \cdots d t=\int_{x_{n}^{0}}^{x_{n}} \cdots \int_{x_{1}^{0}}^{x_{1}} \cdots d t_{1} \cdots d t_{n} .
$$

In fact, under appropriate hypotheses, Young's theorem [18] gives the best possible upper bound, namely

$$
u(x) \leqslant a(x)+\int_{x^{0}}^{x} a(t) b(t) v(t ; x) d t,
$$

where $v(t ; x)$ is the Riemann function relative to the point $x$ for the characteristic initial value problem

$$
\left\{\begin{array}{c}
(-1)^{n} v_{t_{1} \cdots t_{n}}(t ; x)-b(t) v(t ; x)=0 \\
v(t ; x)=1 \quad \text { if } t_{i}=x_{i} \text { for any } i=1,2, \ldots, n
\end{array}\right.
$$

In [13] Snow noted that $v(t ; x)$ is the generalization of an exponential function $\exp \left(\int_{t}^{x} b(r) d r\right)$ which appears in the corresponding bound for a one-dimensional Gronwall inequality (1). The purpose of this note is to give a direct proof of the fact that, when $b(t) \geqslant 0$, the Riemann function $v(t ; x)$ is actually bounded above by this exponential function. This implies, for example, that if also $a \geqslant 0$ in (1), then a more

Received by the editors July 20, 1982.

1980 Mathematics Subject Classification. Primary 26D10; Secondary 45D05, 35B35. 
explicit (if cruder) upper bound for $u$ can be given, namely

$$
u(x) \leqslant a(x)+\int_{x^{0}}^{x} a(t) b(t) \exp \left(\int_{t}^{x} b(r) d r\right) d t .
$$

Such a result, (4), has recently been obtained by the author [2] for a more general inequality than (1), but in a context not involving the Riemann function. Moreover, inequality (5) appears in the middle of a proof [5, Corollary 1] of a matrix version of an extension of (4). (In [5], the argument is also obscured by the fact that the inequality $v \leqslant \exp \left(\int b\right)$ is obtained by applying a form of the main theorem with the inequality sign reversed.) Despite this, the result does not appear to be known. In view of the fact that in most cases where Riemann's method is employed in the cited papers, only an upper bound on $u$ is required (or obtained), it seems to be worthwhile to give a direct proof of the inequality

$$
v(t ; x) \leqslant \exp \left(\int_{t}^{x} b(r) d r\right)
$$

We state the result in the form in which it is most often applicable, with $x^{0}=0$ and $x \geqslant 0$. Here we use the partial order in $\mathbf{R}^{n}: t \leqslant x$ means $t_{i} \leqslant x_{i}$ for $1 \leqslant i \leqslant n$.

THEOREM. Suppose $b: \mathbf{R}^{n} \rightarrow \mathbf{R}$ is continuous and nonnegative for $x \geqslant 0$, and $v(t ; x)$, $0 \leqslant t \leqslant x$, is the Riemann function defined by (3). Then (5) holds for $0 \leqslant t \leqslant x$.

Proof. As noted in [13, (5) and 18, (11)], $v(t ; x)$ is the solution of the integral equation

$$
v(t ; x)=1+\int_{t}^{x} b(s) v(s ; x) d s .
$$

This also follows either by integration of (3) or by differentiation of (6). Moreover, $v(t ; x)$ has the Neumann series representation

$$
v(t ; x)=\sum_{0}^{\infty} u_{k}(t ; x)
$$

where $u_{0}(t ; x)=1$ and, for $k \geqslant 1$,

$$
u_{k}(t ; x)=\int_{t}^{x} b(t) u_{k-1}\left(t_{1} ; x\right) d t_{1}=\cdots=\int_{t}^{x} \int_{t_{1}}^{x} \cdots \int_{t_{k-1}}^{x} \prod_{1}^{k} b\left(t_{i}\right) d t_{k} \cdots d t_{1}
$$

(See also [18] and W. Walter [16, pp. 143-144].) To estimate this integral we use the technique of A. M. Fink [6], as used also by the author in [2]. Set $\tilde{t}=\left(t_{1}, \ldots, t_{k}\right) \in$ $\mathbf{R}^{n k}$, let $\sigma$ denote the set of all $k$ ! permutations of $(1,2, \ldots, k), p \in \sigma$, and

$$
\begin{aligned}
S & =\left\{\tilde{t}: t \leqslant t_{1} \leqslant t_{2} \leqslant \cdots \leqslant t_{k-1} \leqslant t_{k} \leqslant x\right\}, \\
S_{p} & =\left\{\tilde{t}: t \leqslant t_{p_{1}} \leqslant t_{p_{2}} \leqslant \cdots \leqslant t_{p_{k-1}} \leqslant t_{p_{k}} \leqslant x\right\} .
\end{aligned}
$$

Because of the symmetry of the integrand $B(\tilde{t})=\Pi_{1}^{k} b\left(t_{i}\right)$, it follows that

$$
u_{k}(t ; x)=\int_{S} B(\tilde{t}) d \tilde{t}=\int_{S_{p}} B(\tilde{t}) d \tilde{t} \quad \text { for all } p \in \sigma,
$$


whence

$$
u_{k}(t ; x)=\sum_{p \in \sigma} \int_{S_{p}} B(\tilde{t}) d \tilde{t} / k !
$$

If $p, q$ are different permutations in $\sigma$, then $A=S_{p} \cap S_{q}$ has $n k$-dimensional Lebesgue measure 0 since $A$ is a subset of some hyperplane $t_{i}=t_{j}$ of dimension $n(k-1)$. In addition, we clearly have

$$
C=\bigcup_{p \in \sigma} S_{p} \subset[t, x]^{k}=[t, x] \times \cdots \times[t, x],
$$

where $[t, x]=\left\{s \in \mathbf{R}^{n}: t \leqslant s \leqslant x\right\}$. (Note that for $n>1$, " $\subset$ " cannot be replaced by " $=$ " because " $\leqslant$ " is only a partial order in $\mathbf{R}^{n}$.) It now follows that for $0 \leqslant t \leqslant x$

$$
u_{k}(t ; x)=\int_{C} B(\tilde{t}) d \tilde{t} / k ! \leqslant \int_{t}^{x} \cdots \int_{t}^{x} \prod_{1}^{k} b\left(t_{i}\right) d t_{k} \cdots d t_{1} / k !
$$

or

$$
u_{k}(t ; x) \leqslant\left(\int_{t}^{x} b(s) d s\right)^{k} / k ! \text { for } 0 \leqslant t \leqslant x, k=0,1, \ldots
$$

The conclusion (5) now follows from (7) and (9).

More generally, even if $b$ changes sign, it is clear from the above analysis that

$$
|v(t ; x)| \leqslant \exp \left(\int_{t}^{x}|b(s)| d s\right) \text { for } 0 \leqslant t \leqslant x .
$$

This extends the range of applicability of the Riemann method in dealing with integral inequalities (1).

\section{REFERENCES}

1. R. P. Agarwal, On an integral inequality in $n$ independent variables, J. Math. Anal. Appl. 85 (1982), 192-196.

2. P. R. Beesack, On some Gronwall-type integral inequalities in $n$ independent variables, J. Math. Anal. Appl. (to appear).

3. B. K. Bondge and B. G. Pachpatte, On some fundamental integral inequalities in $n$ independent variables, Bull. Inst. Math. Acad. Sinica 8 (1980), 553-560.

4. On some partial integral inequalities in two independent variables, Funkcial. Ekvac. 23 (1980), 327-334.

5. J. Chandra and P. W. Davis, Linear generalizations of Gronwall's inequality, Proc. Amer. Math. Soc. 60 (1976), 157-160.

6. A. M. Fink, Wendroff's inequalities, Nonlinear Anal. 5 (1981), 873-874.

7. D. Y. Kasture and S. G. Deo, Inequalities of Gronwall type in two independent variables, J. Math. Anal. Appl. 58 (1977), 361-372.

8. B. G. Pachpatte, On some new integral and integrodifferential inequalities in two independent variables and their applications, J. Differential Equations 33 (1979), 249-272.

9. __ On some fundamental partial integral inequalities, J. Math. Anal. Appl. 73 (1980), 238-251.

10. On some new integral inequalities for nonselfadjoint hyperbolic partial integrodifferential equations, J. Math. Anal. Appl. 76 (1980), 58-71.

11. . On some partial integral inequalities in $n$ independent variables, J. Math. Anal. Appl. 79 (1981), 256-272.

12. $\ldots$, On certain integral inequalities for partial differential and integral equations, An. Ştiinţ. Univ. “Al. I. Cuza” Iaşi Secţ. I a Mat. (N.S.) 27 (1981), 365-374. 
13. D. R. Snow, A two independent variable Gronwall-type inequality, Inequalities III (Proc. Third Sympos. Univ. California, Los Angeles, Calif., 1969), Academic Press, New York, 1972, pp. 333-340.

14. Gronwall's inequality for systems of partial differential equations in two independent variables, Proc. Amer. Math. Soc. 33 (1972), 46-54.

15. E. Thandapani and R. P. Agarwal, On some new inequalities in $n$ independent variables, J. Math. Anal. Appl. 86 (1982), 542-561.

16. W. Walter, Differential and integral inequalities, Ergebnisse Math. Grenzgeb., Band 55, SpringerVerlag, Berlin and New York, 1970.

17. C.-C. Yeh, On some integral inequalities in $n$ independent variables and their applications, J. Math. Anal. Appl. 86 (1982), 387-410.

18. E. C. Young, Gronwall's inequality in $n$ independent variables, Proc. Amer. Math. Soc. 41 (1973), $241-244$.

Department of Mathematics and Statistics, Carleton University, OtTawa, Ontario, Canada K1S 5B6 